\title{
Evaluating crystallographic likelihood functions using numerical quadratures.
}

\author{
P Zwart ${ }^{1}$, D Perryman ${ }^{2}$ \\ ${ }^{1}$ Molecular Biophysics and Integrated Bioimaging \& \\ Center for Advanced Mathematics for Energy Research Applications \\ Lawrence Berkeley National Laboratories, Berkley, USA ${ }^{2}$ U. Tennessee, Knoxville, TN \\ phzwart@lbl.go
}

Intensity-based likelihood functions in crystallographic applications have the potential to enhance the quality of structures derived from marginal diffraction data. Their usage however is complicated by the ability to efficiently compute these targets functions. Here a numerical quadrature is developed that allows for the rapid evaluation of intensity-based likelihood functions in crystallographic applications. By using a sequence of change of variable transformations, including a non-linear domain compression operation, an accurate, robust, and efficient quadrature is constructed. The approach is flexible and can incorporate different noise models with relative ease. 1. Zwart, P.H. \& Perryman, D. E. Under review. https://bit.ly/3dVOuQS; 2. Murshudov, G. N., Vagin, A. A. \& Dodson, E. J. (1997). Acta Crystallogr D Biol Crystallogr, 53(Pt 3), 240-55; 3. Pannu, N. S. \& Read, R. J. (1996). Acta Crystallographica Section A Foundations of Crystallography, 52(5), 659-668; 4. de La Fortelle, E. \& Bricogne, G. (1997). In Macromolecular Crystallography Part A, vol. 276 of Methods in Enzymology, pp. 472 - 494.

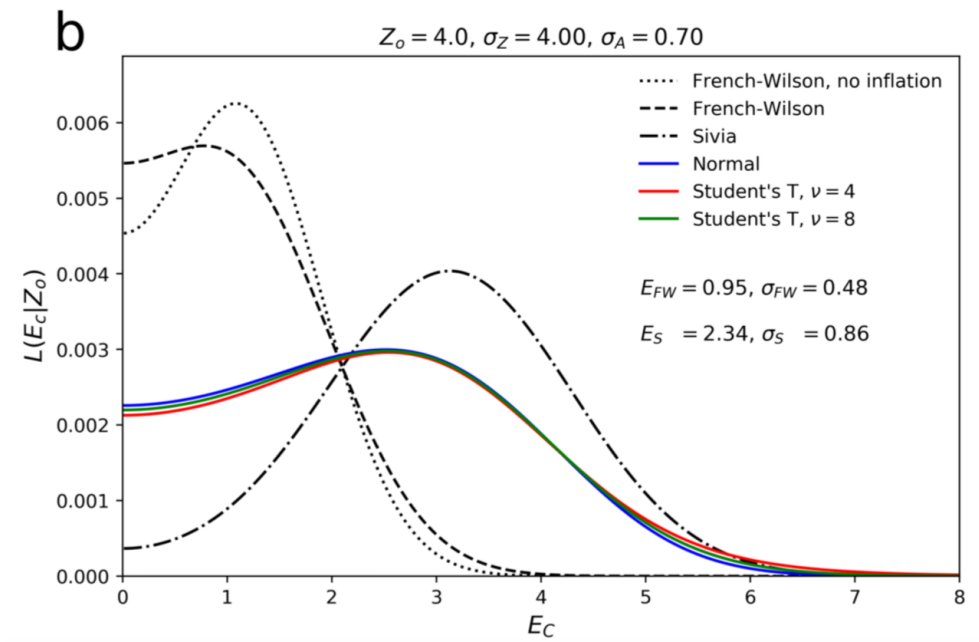

Figure 1. A sample likelihood function computed using a 49-point quadrature method under the assumption of normal and Student's t-distributed noise with different degrees of freedom, is compared to French-Wilson-like

Acta Cryst. (2020). A76, a60 